\title{
Genetic parameters of direct and maternal effects for calving ease in Dutch Holstein-Friesian cattle
}

\author{
S. A. E. Eaglen and P. Bijma ${ }^{1}$ \\ Animal Breeding and Genomics Centre, Wageningen University, Marijkeweg 40, 6709PG, Wageningen, the Netherlands
}

\section{ABSTRACT}

Genetic parameters of direct and maternal effects for calving ease in Dutch dairy cattle were estimated using 677,975 calving ease records from second calving. Particular emphasis was given to the presence and impact of environmental dam-offspring covariances on the estimated direct-maternal genetic correlation. Moreover, a measure of heritability for traits affected by maternal effects was developed. In contrast to previous parameters, this parameter reflects the amount of genetic variance that can be used to generate a response to selection in maternally affected traits. Estimated genetic correlations between direct and maternal effects on calving ease have often been moderately negative, particularly in beef cattle. Environmental dam-offspring covariances have been put forward as an explanation for such estimates. We investigated the impact of environmental dam-offspring covariances by fitting correlated residuals between dam and offspring records in the statistical model, and by comparing results of a sire-maternal grandsire model with those of an animal model. Results show that calving ease in Dutch dairy cattle has a direct heritability of approximately 0.08 , a maternal heritability of approximately 0.04 , a directmaternal genetic correlation of approximately -0.20 , and a total heritable variance equal to approximately $11 \%$ of phenotypic variance. Results of animal models and sire-maternal grandsire models were very similar. The direct-maternal environmental covariance was near zero, and consequently had very little impact on the estimated genetic parameters. Transformation of observations to a liability scale did not affect the estimated genetic parameters and yielded a nearly identical ranking of sires.

Key words: calving ease, genetic parameter, maternal genetic effect, direct-maternal genetic correlation

Received August 26, 2008.

Accepted December 9, 2008.

${ }^{1}$ Corresponding author: piter.bijma@wur.nl
INTRODUCTION

Calving ease is of great importance to the dairy industry (Luo et al., 2002; Hickey et al., 2007). In addition to having economic effects, it affects animal welfare, with dystocia being ranked as one of the most painful conditions of cattle (Huxley and Whay, 2006). Calving ease is determined by the calf as well as by its dam, respectively, through effects termed direct and maternal. From the standpoint of the offspring, the maternal effect is purely environmental. However, it has a genetic component and thus could contribute to selection response (Dickerson, 1947; Willham, 1963).

In beef cattle, estimates of the direct-maternal genetic correlation have often been moderately negative and have therefore been doubted (Robinson 1996). Koch (1972) remarked that ignoring a possible environmental dam-offspring covariance may cause a bias when estimating the genetic direct-maternal correlation. Falconer (1965) suggested fitting a partial regression coefficient on the phenotype of the dam to account for such environmental covariance. The model of Falconer (1965), however, yielded biased estimates when simulated or when integrated with the model of Willham (1963; Robinson, 1996; Koerhuis and Thompson, 1997). In a study on broiler chickens, Koerhuis and Thompson (1997) presented an extension of the model of Willham (1963) to account for environmental dam-offspring correlations by fitting a covariance between the commonenvironmental effect and the residual of the model. For species in which litters consist of a single individual, such as cattle, Bijma (2006) suggested fitting correlated residuals rather than regression on the maternal phenotype to account for environmental dam-offspring covariances. In a simulation study, this model yielded unbiased estimates. Studies investigating the effect of environmental dam-offspring covariances on genetic parameters of calving ease have been limited to beef cattle (Robinson, 1996). We could not find similar studies in dairy cattle.

The first objective of this work was to investigate the presence and consequences of an environmental damoffspring covariance on calving ease in dairy cattle. We estimated genetic parameters for direct and maternal 
effects on calving ease in Dutch Holstein-Friesian cattle, and investigated the impact of a potential environmental dam-offspring covariance on the estimated genetic parameters.

A second objective was to investigate the meaning of heritability and total heritable variance in traits affected by maternal effects. Several studies have presented a "total heritability," referring to Willham (1972). This total heritability, however, refers strictly to the response to mass selection (Dickerson, 1947; Willham 1963, 1972), which is rare in modern livestock genetic improvement. We present a measure of heritable variance that represents the magnitude of genetic differences that can be used to generate a response to selection, irrespective of the selection method.

\section{MATERIALS AND METHODS}

\section{Heritable Variance with Maternal Effects}

In the absence of maternal effects, the interpretation of additive genetic variance and heritability is unambiguous. In that case, phenotypes are the sum of an additive genetic effect (the breeding value) and a nonheritable component, $P=A+E$. Heritability equals the proportion of the phenotypic variance originating from additive genetic effects, $h^{2}=\operatorname{Var}(A) / \operatorname{Var}(P)$. Response to mass selection is the product of heritability and the selection differential, $R=h^{2} S$. In addition, response for any selection strategy is the product of intensity, accuracy, and additive genetic standard deviation, $R=\iota \rho \sigma_{A}$, indicating that $\sigma_{A}$ represents the magnitude of the genetic differences that can be used to generate a response to selection. With maternal effects, however, there is a difference between the proportion of phenotypic variance attributable to additive genetic effects, the realized heritability of mass selection, and the magnitude of the genetic differences that can be used to generate a response to selection. Because genetic improvement is the ultimate goal in animal breeding, we concentrate on the magnitude of the genetic differences that can be used to generate a response to selection.

Following Dickerson (1947) and Willham (1963), we model the phenotype of individual $i$ as

$$
P_{i}=A_{D, i}+E_{D, i}+A_{M, j}+E_{M, j},
$$

where $i$ is the individual, $j$ is its dam, $A_{D}$ and $A_{M}$ are the direct and maternal breeding values, and $E_{D}$ and $E_{M}$ are the nonheritable direct and maternal effects. From equation [1], it follows that response to selection is the sum of the increases in the mean direct and maternal breeding values (Dickerson, 1947; Willham, 1963, 1972),

$$
R=\Delta \bar{A}_{D}+\bar{\Delta} A_{M}
$$

which is a direct analogy of $R=\Delta \bar{A}$ in the absence of maternal effects. From equation [2], it follows that the total breeding value (TBV) of an individual (i.e., the value of the individual for genetic improvement) is the sum of its direct and maternal breeding value,

$$
T B V_{i}=A_{D, i}+A_{M, i} .
$$

In contrast to the phenotypic value, the TBV includes the individual's own breeding value for a maternal effect, rather than that of its dam. This is because an individual transmits its own breeding value for maternal effects to its offspring, not that of its dam. The TBV in equation [3] represents the heritable impact of an individual on the mean trait value of the population, $\bar{P}=\bar{A}_{D}+\bar{A}_{M}$. The TBV, therefore, properly summarizes the "value of an individual for breeding." From equation [3], it follows that the total heritable variance that can be used to generate a response to selection equals the variance in TBV among individuals,

$$
\sigma_{T B V}^{2}=\sigma_{A_{D}}^{2}+2 \sigma_{A_{D M}}+\sigma_{A_{M}}^{2} .
$$

By analogy to the situation without maternal effects, response to selection equals

$$
R=\iota \rho_{M} \sigma_{T B V},
$$

where $\rho_{M}$ is the accuracy of selection for maternally affected traits, which is the correlation between the selection criterion and the TBV of an individual. The above reasoning is somewhat intuitive; a formal derivation of equation [5] is shown in the Appendix.

To express total heritable variance on the scale of heritability, we defined $T^{2}$ as the ratio of heritable variance over phenotypic variance,

$$
T^{2}=\frac{\sigma_{T B V}^{2}}{\sigma_{P}^{2}} .
$$

For example, $T^{2}=0.10$ indicates that the heritable variance that can be used for genetic improvement equals $10 \%$ of the phenotypic variance.

The total heritable variance defined in equation [4] differs from the genetic component of phenotypic variance. Phenotypic variance (Willham, 1963, 1972) equals

$$
\sigma_{P}^{2}=\sigma_{A_{D}}^{2}+\sigma_{A_{D M}}+\sigma_{A_{M}}^{2}+\sigma_{E_{D}}^{2}+\sigma_{E_{M}}^{2} .
$$


Hence, the genetic component of phenotypic variance is

$$
\operatorname{Var}_{g}(P)=\sigma_{A_{D}}^{2}+\sigma_{A_{D M}}+\sigma_{A_{M}}^{2} .
$$

Willham (1972) referred to this amount as the total genetic variance. Comparison of equations [4] and [8] shows that there is a difference, $\sigma_{A_{D M}}$, between the total genetic variance, $\operatorname{Var}_{g}(P)$, and the heritable variance that can be used for genetic improvement, $\sigma_{T B V}^{2}$.

Several studies have considered a "total heritability," referring to Willham (1972), Meyer (1992), Koch et al. (1994), and Luo et al. (2002),

$$
h_{r}^{2}=\frac{\sigma_{A_{D}}^{2}+1 \frac{1}{2} \sigma_{A_{D M}}+\frac{1}{2} \sigma_{A_{M}}^{2}}{\sigma_{P}^{2}} .
$$

Willham $(1963,1972)$, however, referred to $h_{r}^{2}$ as the "fraction of the selection differential realized if selection were on $P_{x}$," rather than "total heritability." Hence, $h_{r}^{2}$ represents the realized heritability for mass selection, $h_{r}^{2}=R / S$. It is the regression coefficient of an individual's TBV on its phenotype, $h_{r}^{2}=b_{T B V, P}=\operatorname{Cov}\left(T B V_{i}, P_{i}\right) / \sigma_{P}^{2}$ (Dickerson, 1947; equation [3]). Hence, the numerator of $h_{r}^{2}$ represents the covariance between TBV and phenotypes of individuals, rather than the total heritable variance.

The above has shown that, with maternal effects, there is a difference between "the proportion of phenotypic variance attributable to additive genetic effects," $\operatorname{Var}_{g}(P) / \sigma_{P}^{2}$; "the realized heritability of mass selection," $h_{r}^{2}$; and "the ratio of total heritable variance available for response to selection over phenotypic variance," $T^{2}=\sigma_{T B V}^{2} / \sigma_{P}^{2}$. The $T^{2}$ seems to be the most relevant for livestock species in which mass selection is rare, such as dairy cattle. In the following, therefore, we also present estimates of $T^{2}$.

\section{Data}

A data set consisting of 872,918 calving ease records was provided by the Royal Dutch Cattle Syndicate (NRS). Until recently, calving ease in the Netherlands was recorded only for offspring of young bulls. Young bulls are used on first-lactation cows, so that their calves are born to second-parity cows. At present, very few records are available for first-parity cows. The calving ease records used in this study are therefore all from the second calving. Note that dystocia is usually greater with heifers, so that comparison of our results with those found in heifers may require caution. These records were collected by farmers from 1993 through 2004, who subjectively scored calving ease using a 4 -category scale, where 1 = easy birth, $2=$ normal birth, $3=$ difficult birth, $4=$ very difficult birth (help of a veterinarian). Hence, higher scores indicate greater calving difficulty, although the trait usually is referred to as calving ease rather than calving difficulty. Because farm size is fewer than 100 cows, unobserved calvings are relatively rare in the Netherlands. Farmers were not specifically instructed on how to record calving ease. For all records, 5 generations of pedigree records were available.

From the original data set, twin calvings were discarded. Age at calving, birth weight, parity, and breed were checked for inconsistencies and erroneous records were removed. Sires with fewer than 20 progeny records and herds with fewer than 2 records were removed. Average gestation length equaled 279 d. Records with gestation length outside the range of 260 to $290 \mathrm{~d}$ were removed because there were very few records fewer than $260 \mathrm{~d}$, whereas records exceeding $290 \mathrm{~d}$ may have resulted from a subsequent insemination. The age of the dams was restricted to between 34 and 48 mo because the percentage of cows outside these ages was relatively small, and those cows may have had an error in their parity record. Records of calves sired by bulls with a breed composition other than Holstein-Friesian were removed. Records of cows with a breed composition including beef cattle breeds were removed, but records of cows carrying genes from the dual-purpose breed Meuse-Rhine-Yssel were retained. After editing, the data consisted of 677,795 records originating from 19,985 herds. Table 1 has the breed composition of the cows in the final data, whereas Table 2 has the frequencies of each class of calving ease. There were 4,635 sires represented in the data, all young bulls, and 18,467 maternal grand sires (Table 3 ).

\section{Statistical Methods}

Environmental covariances between records of dam and offspring occur only when the data include both the record of the dam and the record of her offspring. In other words, such environmental covariances occur only for animals recorded as being born as well as animals recorded as giving birth. This occurred only in a subset of the data. We therefore split the data into a subset containing all the animals recorded as being born as well as recorded as giving birth (subset 1, $\mathrm{n}=$ 98,162; Table 3), and a remaining subset without such animals $(\mathrm{n}=579,633)$. 
Table 1. Breed compositions ${ }^{1}$ of dams in the complete data set

\begin{tabular}{lc}
\hline Breed composition $^{2}$ & Records, $\mathrm{n}$ \\
\hline HF8 & 287,629 \\
HF7FH1 & 204,371 \\
HF6FH2 & 78,566 \\
HF7MRY1 & 35,109 \\
HF6MRY2 & 29,938 \\
HF6MRY1 & 4,063 \\
HF6FH1 & 711 \\
HF5FH3 & 2 \\
HF5FH2 & 1 \\
HF4FH4 & 1 \\
Other & 37,404 \\
\hline
\end{tabular}

${ }^{1} \mathrm{HF}=$ Holstein-Friesian; $\mathrm{FH}=$ Fries-Hollands; MRY = Meuse-RhineYssel. Other $=$ all compositions containing HF together with dairy breeds other than FH and MRY.

${ }^{2}$ Indicates proportion of genes of each breed; for example, HF7FH1 = $7 / 8 \mathrm{HF}$ and $1 / 8 \mathrm{FH}$ genes.

We investigated the impact of environmental damoffspring covariances on the estimated genetic parameters in 2 ways. First, we analyzed subset 1 using an animal model that either accounted for environmental dam-offspring covariances or ignored such covariances. Second, we compared results of a sire-maternal grandsire model (S-MGS) applied to subset 1 with results of an animal model. An S-MGS model does not use the relationships between dams and their offspring and should therefore not suffer from environmental damoffspring covariances.

The data set without animals recorded as being born as well as animals giving birth was too large to allow analysis with an animal model. To enable analysis with an animal model, we analyzed several subsets of this data set (subsets 2 to 5; Table 3). Subsets 2 to 5 were created by selecting several herds and subsequently removing records of sires with fewer than 20 progeny, so that the total number of observations in each subset was approximately 100,000. In all cases, variance components were estimated by REML, using ASREML version 2.0 (Gilmour et al., 2002).

Animal Model. The animal model was

$$
\mathbf{y}=\mathbf{X b}+\mathbf{Z}_{D} \mathbf{a}_{D}+\mathbf{Z}_{M} \mathbf{a}_{M}+\mathbf{e},
$$

where $\mathbf{y}$ is a vector of calving ease scores; $\mathbf{X}, \mathbf{Z}_{D}$, and $\mathbf{Z}_{M}$ are known incidence matrices for nongenetic correction factors and for direct and maternal genetic effects; $\mathbf{b}$ is a vector of nongenetic correction factors; $\mathbf{a}_{D}$ is a vector of random direct genetic effects of the calves; $\mathbf{a}_{M}$ is a vector of random maternal genetic effects of the cows, and $\mathbf{e}$ is a vector of residuals. The $\mathbf{a}_{D}$ and $\mathbf{a}_{M}$ were assumed to follow a multivariate normal distribution with mean $\mathbf{0}$ and covariance matrix $\mathbf{G} \otimes \mathbf{A}$, where

$$
\mathbf{G}=\left[\begin{array}{cc}
\sigma_{A_{D}}^{2} & \sigma_{A_{D} A_{M}} \\
\sigma_{A_{D} A_{M}} & \sigma_{A_{M}}^{2}
\end{array}\right],
$$

$\otimes$ indicates the Kronecker product of matrices, and A is the relationship matrix. When ignoring the dam-offspring environmental covariance, residuals were assumed to be independent, $\mathbf{e} \sim N\left(0, \mathbf{I} \sigma_{e}^{2}\right)$, where $\mathbf{I}$ denotes an identity matrix. Nongenetic correction factors in $\mathbf{b}$ included herd, the interaction of year and month of calving, sex of the calf, breed composition of the dam (Table 2), age of the dam in months, and herd-year fitted as a normally distributed random effect. Table 4 shows the distribution of the levels of the fixed effects. The number of subclasses in the full data set, subset 1 , and subset 2 were 135, 128, and 130 for year-season and $132,244,54,261$, and 23,211 for herd-year, respectively.

The presence of an environmental dam-offspring covariance creates a covariance between the residuals of the record of an individual being born and the record of that same individual giving birth (Koch, 1972; Robinson, 1996; Koerhuis and Thompson, 1997; Bijma, 2006). The nongenetic direct effect of the individual is expressed in its birth record, whereas its nongenetic maternal effect is expressed in its calving record. From equation [1], the covariance between the birth and calving record of individual $i$ equals

$$
\begin{gathered}
\operatorname{Cov}\left(P_{i}, P_{j}\right)= \\
\frac{1}{2} \sigma_{A_{D}}^{2}+1 \frac{1}{4} \sigma_{A_{D M}}+\frac{1}{2} \sigma_{A_{M}}^{2}+\operatorname{Cov}\left(E_{D, i}, E_{M, i}\right),
\end{gathered}
$$

where $P_{i}$ represents the birth records of individual $i$ and $P_{j}$ represents the birth record of its offspring $j$ (which is the same record as the calving record of $i$ ). Hence, the nongenetic covariance between dam and offspring record is equal to the environmental direct-maternal covariance, $\sigma_{E_{D M}}$. We accounted for this covariance in the animal model by allowing the residuals of dam and offspring records to be correlated. Thus, when accounting for an environmental dam-offspring covariance, we fitted the residual variance structure $\mathbf{e} \sim N\left(0, \mathbf{R} \sigma_{e}^{2}\right)$, so

Table 2. Frequency distribution of calving ease scores ${ }^{1}$

\begin{tabular}{lc}
\hline Score $^{2}$ & Frequency, \% \\
\hline 1 & 42.07 \\
2 & 50.17 \\
3 & 7.46 \\
4 & 0.29 \\
\hline
\end{tabular}

${ }^{1}$ Frequencies refer to the full data set (Table 3 ).

${ }^{2} 1=$ easy; $2=$ normal; $3=$ difficult; $4=$ very difficult. 
Table 3. Descriptive statistics of the data

\begin{tabular}{lrrr}
\hline Variable & Full & Subset $1^{1}$ & Subset $2^{2}$ \\
\hline Records, n & 677,795 & 98,162 & 99,328 \\
Sires, n & 4,635 & 4,586 & 2,683 \\
Herds, n & 19,985 & 11,363 & 4,055 \\
Dams, n & 677,795 & 98,162 & 99,328 \\
Dams with own birth record, n & 101,445 & 49,081 & 0 \\
Maternal grandsires, n & 18,467 & 6,474 & 17,963 \\
\hline
\end{tabular}

${ }^{1}$ Subset 1 included all individuals recorded at birth as well as individuals giving birth.

${ }^{2}$ Subsets 3 through 5 were very similar to subset 2, each containing approximately 100,000 records, obtained by selecting all records from a subset of the herds. Subsets did not have overlapping records.

that $\operatorname{Var}(\mathbf{e})=\mathbf{R} \sigma_{e}^{2}$, where elements of $\mathbf{R}$ are given by $R_{i i}$ $=1, R_{i j}=\rho$ when $i$ and $j$ are dam and offspring, and $R_{i j}=0$ otherwise (Bijma, 2006). This variance structure was fitted by using the CORU statement in the Rstructure definition in ASREML version 2.0 (Gilmour et al., 2002). Because combined dam-offspring records occurred only in subset 1 , this model was fitted to subset 1 only.

\section{S-MGS Model. The S-MGS model was}

$$
\mathbf{y}=\mathbf{X b}+\mathbf{Z}_{S} \mathbf{a}_{S}+\mathbf{Z}_{M G S} \mathbf{a}_{M G S}+\mathbf{e},
$$

where $\mathbf{Z}_{S}$ and $\mathbf{Z}_{M G S}$ are known incidence matrices, $\mathbf{a}_{S}$ is a vector of random additive genetic effects of the sires of the calves, $\mathbf{a}_{M G S}$ is a vector of the random additive genetic effects of the maternal grandsires of the calves, and $\mathbf{a}_{S}$ and $\mathbf{a}_{M G S}$ are assumed to follow a multivariate normal distribution with mean $\mathbf{0}$ and covariance matrix $\mathbf{G} \otimes \mathbf{A}$ where

$$
\mathbf{G}=\left[\begin{array}{cc}
\sigma_{A_{S}}^{2} & \sigma_{A_{S} A_{M G S}} \\
\sigma_{A_{S} A_{M G S}} & \sigma_{A_{M G S}}^{2}
\end{array}\right]
$$

and $\mathbf{A}$ is the relationship matrix specific for the S-MGS model. Correction factors in $\mathbf{b}$ were the same as for the animal model. Variance components estimated from the S-MGS model were converted into direct and maternal variance components using $\quad \sigma_{A_{D}}^{2}=4 \sigma_{A_{S}}^{2}, \quad \sigma_{A_{D M}}=4 \sigma_{A_{S} A_{M G S}}-2 \sigma_{A_{S}}^{2}, \quad$ and $\sigma_{A_{M}}^{2}=4 \sigma_{A_{M G S}}^{2}+\sigma_{A_{S}}^{2}-4 \sigma_{A_{S} A_{M G S}}$ (Steinbock et al., 2002; Wiggans et al., 2003).

Transformed $y$-Values. Categorical traits are often believed to be the result of an underlying normally distributed liability. In this model, the liability is an unobservable variable, and the phenotypes are the result of the position of the liability with respect to some thresholds separating the observed categories. This model is known as a threshold model (Wright, 1934). To better account for the categorical nature of the calving ease scores, the NRS transforms the recorded calving ease scores $(1,2,3$, and 4$)$ into the average liability value corresponding to the observed phenotypic category. The transformed values are fully determined by the frequencies of the categories (Table 2). To investigate the effect of such a transformation, we also estimated genetic parameters and breeding values using transformed calving ease scores. Based on the frequency of each category (Table 2), the transformed values of categories 1 through 4 are $-0.931,0.517,1.866$, and 3.047. Beware that this transformation does not make calving ease scores continuous; it merely alters the differences between scores.

Table 4. Distribution of data for each level of fixed effects

\begin{tabular}{lcc}
\hline Variable & Frequency, $\%$ & Observations, $\mathrm{n}$ \\
\hline Birth month of calf & & \\
January & 8.3 & 56,251 \\
February & 8.0 & 54,019 \\
March & 8.5 & 57,713 \\
April & 6.9 & 46,985 \\
May & 6.1 & 41,480 \\
June & 5.7 & 38,661 \\
July & 7.0 & 47,382 \\
August & 10.2 & 69,236 \\
September & 10.2 & 69,034 \\
October & 10.2 & 69,124 \\
November & 9.5 & 64,089 \\
December & 9.4 & 63,821 \\
Sex of calf & & \\
Male & 51.2 & 346,677 \\
Female & 48.9 & 331,118 \\
Birth year of calf & & \\
1993 & 2.0 & 13,801 \\
1994 & 8.0 & 54,064 \\
1995 & 9.1 & 61,866 \\
1996 & 9.3 & 63,319 \\
1997 & 8.4 & 56,930 \\
1998 & 10.6 & 71,751 \\
1999 & 11.3 & 76,796 \\
2000 & 10.9 & 73,990 \\
2001 & 9.5 & 64,696 \\
2002 & 8.3 & 56,034 \\
2003 & 8.0 & 54,136 \\
2004 & 4.5 & 30,412 \\
\hline
\end{tabular}




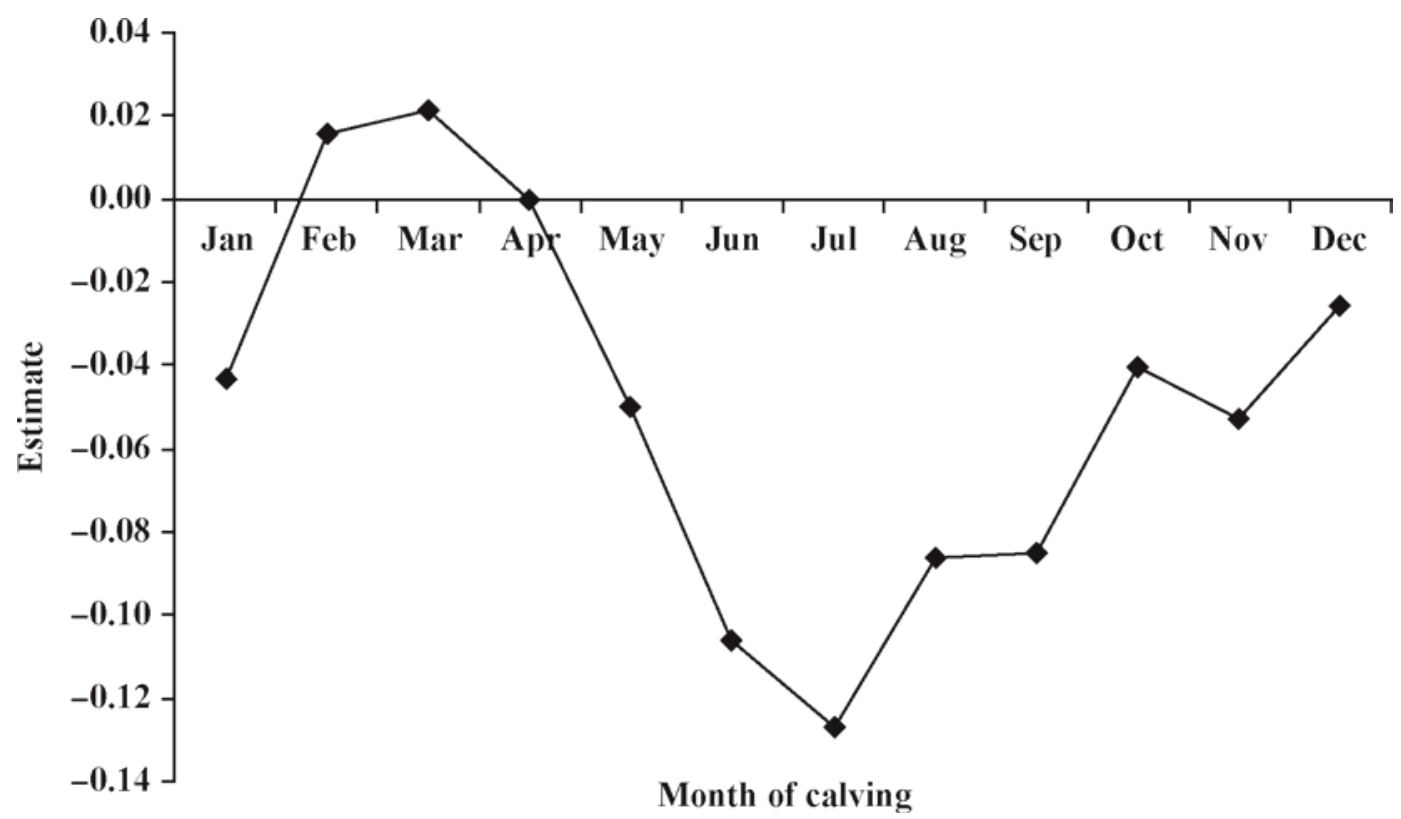

Figure 1. Effect of month of calving on calving difficulty, expressed relative to April.

\section{RESULTS AND DISCUSSION}

\section{Fixed Effects}

Solutions for fixed effects indicated more difficult calvings in winter, which agrees with previous findings (Figure 1; Manfredi et al., 1991; Steinbock et al., 2002). When compared with female calves, male calves had $0.2536 \pm 0.0014$ greater calving difficulty, which is likely attributable to a difference in body size and shape (Hickey et al., 2007). Calving difficulty increased with age of the cow (Figure 2; note that all records were from the second parity). To clarify the origin of this effect, we investigated the relationship between gestation length and age at calving and the impact of gestation length on calving ease. When fitting gestation length as a covariate in the model, the effect of age on calving ease decreased by approximately $75 \%$. This shows that approximately $75 \%$ of the age effect was related to longer gestations in older cows. Mean gestation length was $2.3 \mathrm{~d}$ longer in cows calving at 48 mo of age compared with cows calving at 34 mo of age, and longer gestation was related to greater calving difficulty. Thus, increased gestation length seems to be an important cause of increased calving difficulty at higher ages. (Note that gestation length was not included in the model used for estimating genetic parameters.) The age at calving was not related to breed composition (Table 5). Moreover, the increase of calving difficulty with age may be related to low fertility, which is associated with both extended calving intervals, and thus greater age at calving, and more calving difficulty
(Mee, 2007). Breed composition of the dam had a small but significant effect on calving ease. Combinations of the Holstein-Friesian and the Meuse-Rhine-Yssel breed showed easier calving, followed by purebred HolsteinFriesian and the combination of Holstein-Friesian with the Fries-Holland breed.

\section{Genetic Parameters}

Table 6 provides estimated genetic parameters. All estimated heritabilities differed significantly from zero $(P<0.05)$. On average, direct heritabilities were approximately twice as large as maternal heritabilities $(\sim 0.08$ vs. $\sim 0.04)$. Estimated heritabilities were in the range of previously published estimates on calving ease in dairy cattle (all parities), which ranged from 0.072 to 0.13 for direct heritability and from 0.007 to 0.07 for maternal heritability (Cue and Hayes, 1985; Djemali et al., 1987; Weller and Gianola., 1989; Manfredi et al., 1991; Weller and Ron, 1992; Luo et al., 2002; Wiggans et al., 2003; Hickey et al., 2007). Note that parity definition differed among studies. Because first-parity calving ease was not used in our data, we could not account for selection in the data because of culling after extreme difficulty in first calving.

Average heritabilities from animal model analyses of subsets 2 through 5 were very similar to results of the S-MGS analysis of all data. The relatively large differences between estimated maternal heritabilities from subsets 2 through 5 indicate that accurate estimation of $h_{M}^{2}$ is difficult, even with approximately 100,000 records. 


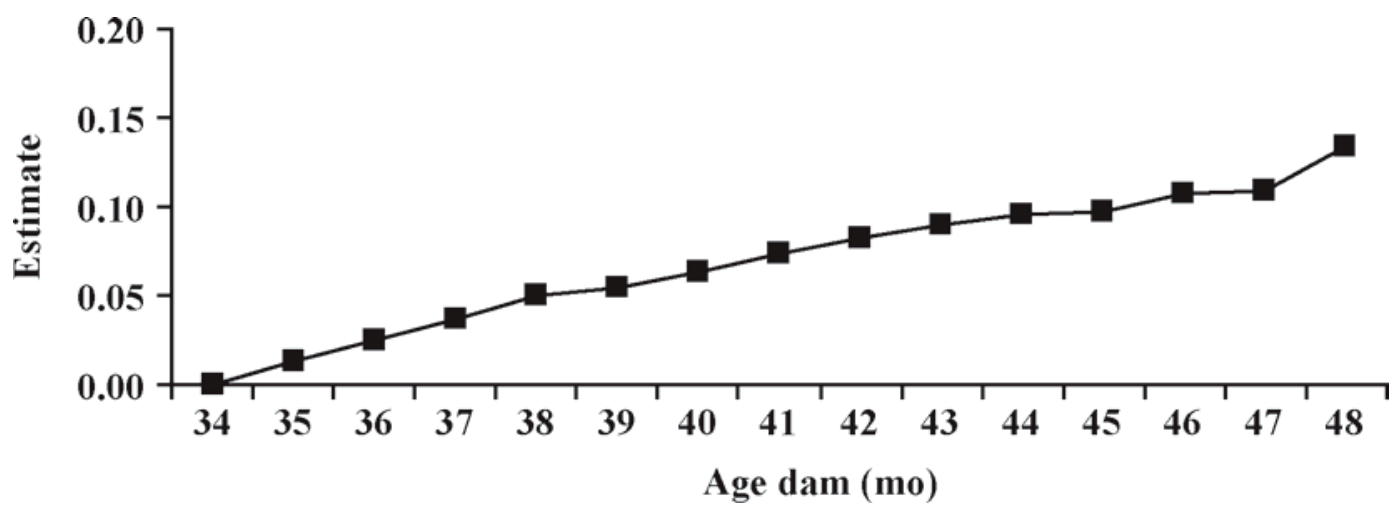

Figure 2. Effect of age of dam on calving difficulty, expressed relative to an age of 34 mo.

Estimated direct-maternal genetic correlations were negative in all cases, ranging from -0.04 through -0.44 . The average direct-maternal genetic correlation from animal model analyses of subsets 2 through 5 was somewhat more negative than the result of the S-MGS analysis of the full data $(-0.24 \pm 0.06$ vs. $-0.15 \pm 0.04)$. The relatively large differences between estimated direct-maternal genetic correlations from subsets 2 through 5 indicate that accurate estimation of $r_{G_{D M}}$ is also difficult, even with approximately 100,000 records. Other studies of calving ease in dairy cattle reported similar correlations, ranging from -0.07 to -0.47 (Thompson et al., 1981; Cue and Hayes, 1985; Meijering and Postma, 1985; Manfredi et al., 1991; Wiggans et al., 2003; Hickey et al., 2007). Analysis of beef cattle data often yields estimates that are more negative (Robinson, 1996).

Total heritable variance of calving ease, expressed relative to phenotypic variance $\left(T^{2}\right)$, ranged from 0.085 through 0.120 , and averaged 0.107 for both animal model analysis of subsets 2 through 5 and S-MGS

Table 5. Estimated effects of breed composition categories ${ }^{1}$

\begin{tabular}{lrc}
\hline Breed composition $^{2}$ & Records, $\mathrm{n}$ & Estimate \\
\hline HF8 & 287,629 & 0 \\
HF7FH1 & 204,371 & 0.00784 \\
HF6FH2 & 78,566 & 0.00658 \\
HF7MRY1 & 35,109 & 0.00504 \\
HF6MRY2 & 29,938 & 0.00622 \\
HF6MRY1 & 4,063 & -0.00549 \\
HF6FH1 & 711 & 0.01127 \\
HF5FH3 & 2 & -0.3488 \\
HF5FH2 & 1 & 0.3951 \\
Rest & 37,404 & 0.00053 \\
\hline
\end{tabular}

${ }^{1} \mathrm{HF}=$ Holstein Friesian; FH = Fries-Hollands; MRY = Meuse-RhineYssel; Rest $=$ all compositions containing HF together with dairy breeds other than FH and MRY.

${ }^{2}$ Indicates proportion of genes of each breed; for example, HF7FH1 = $7 / 8 \mathrm{HF}$ and $1 / 8 \mathrm{FH}$ genes. analysis of all data. A comparison of this result with the estimated direct heritability showed that the presence of maternal effects increased heritable variance by approximately $25 \%$.

Transforming observed calving scores to a liability scale affected neither the estimated heritabilities and genetic correlations nor their standard errors. Moreover, EBV and rankings of sires and maternal grandsires on their TBV correlated with a Pearson and rank correlation coefficient of $>0.99$ between the 2 analyses. Transformation to a liability scale, therefore, would lead to the same selection decisions in a breeding program, and thus the same response to selection.

Heritabilities estimated from subset 1 were somewhat lower than the average estimates, although the difference was small and nonsignificant. This difference may originate from selection in the data. Subset 1 contained only the animals recorded as being born as well as animals giving birth. Thus, the animals recorded as being born must have been $100 \%$ females, and they have given birth to approximately 50\% male and approximately $50 \%$ female calves. Consequently, subset 1 consisted of approximately $75 \%$ female calves and approximately $25 \%$ male calves. Because females are usually born easier, this selection reduced the incidence of dystocia in subset 1, which may have lowered the estimated heritabilities somewhat.

The analysis of subset 1 allowing for correlated residuals of dam and offspring records yielded a very small and nonsignificant residual correlation of $0.003 \pm$ 0.008 . This residual correlation is equal to the ratio of the environmental dam-offspring covariance and the residual variance, $\rho=\sigma_{E_{D M}} / \sigma_{e}^{2}$ (equation [11] and below). Hence, the estimated residual correlation of approximately 0 indicates that environmental dam-offspring covariances are practically absent in calving ease in Dutch dairy cattle. This conclusion is supported by the small difference between the direct-maternal ge- 
Table 6. Estimated genetic parameters ${ }^{1}$

\begin{tabular}{|c|c|c|c|c|c|}
\hline \multirow[b]{2}{*}{ Model and data set } & \multicolumn{5}{|c|}{ Parameter $^{2}$} \\
\hline & $h_{D}^{2}$ & $h_{M}^{2}$ & $r_{G_{D M}}$ & $\rho$ & $T^{2}$ \\
\hline \multicolumn{6}{|l|}{ Animal model } \\
\hline Subset 1 , independent residuals ${ }^{3}$ & $0.077 \pm 0.007$ & $0.028 \pm 0.006$ & $-0.11 \pm 0.09$ & - & $0.095 \pm 0.007$ \\
\hline Subset 1 , correlated residuals ${ }^{4}$ & $0.076 \pm 0.008$ & $0.027 \pm 0.006$ & $-0.13 \pm 0.11$ & $0.003 \pm 0.008$ & $0.091 \pm 0.012$ \\
\hline Subset 2 & $0.088 \pm 0.007$ & $0.042 \pm 0.010$ & $-0.10 \pm 0.14$ & - & $0.116 \pm 0.013$ \\
\hline Subset 3 & $0.087 \pm 0.007$ & $0.055 \pm 0.011$ & $-0.16 \pm 0.12$ & - & $0.120 \pm 0.014$ \\
\hline Average subsets 2 to 5 & $0.088 \pm 0.004$ & $0.040 \pm 0.005$ & $-0.24 \pm 0.06$ & - & $0.107 \pm 0.006$ \\
\hline \multicolumn{6}{|l|}{ Sire-maternal grandsire model } \\
\hline Subset 1 & $0.075 \pm 0.006$ & $0.020 \pm 0.005$ & $-0.07 \pm 0.12$ & - & $0.089 \pm 0.009$ \\
\hline Full data & $0.084 \pm 0.003$ & $0.041 \pm 0.004$ & $-0.15 \pm 0.04$ & - & $0.107 \pm 0.005$ \\
\hline Full data, transformed observations ${ }^{5}$ & $0.083 \pm 0.003$ & $0.040 \pm 0.004$ & $-0.15 \pm 0.04$ & - & $0.107 \pm 0.005$ \\
\hline
\end{tabular}

${ }^{1}$ Phenotypic variance was 0.32 for the full data, 0.30 for subset 1 , approximately 0.32 for subsets 2 through 5 , and 0.65 with transformed y-values. The greater phenotypic variance with transformed y-values has no biological meaning; it merely indicates a difference in scale of the records. Subset 1 included all individuals occurring both as offspring and as dam; subsets 2 to 5 included no such individuals.

${ }^{2} h_{D}^{2}=\sigma_{A_{D}}^{2} /\left(\sigma_{A_{D}}^{2}+\sigma_{A_{D M}}+\sigma_{A_{M}}^{2}+\sigma_{e}^{2}\right) ; h_{M}^{2}=\sigma_{A_{M}}^{2} /\left(\sigma_{A_{D}}^{2}+\sigma_{A_{D M}}+\sigma_{A_{M}}^{2}+\sigma_{e}^{2}\right) ; \rho$ is the estimated correlation between the residuals of dam and offspring records; and $T^{2}=\sigma_{T B V}^{2} / \sigma_{P}^{2}$, where TBV denotes the total breeding value (equations [3] and [4]).

${ }^{3}$ Residuals were assumed to be independent, $\mathbf{e} \sim N\left(0, \mathbf{I} \sigma_{e}^{2}\right)$.

${ }^{4}$ Residuals of dam and offspring records were allowed to be correlated, $\mathbf{e} \sim N\left(0, \mathbf{R} \sigma_{e}^{2}\right)$.

${ }^{5}$ Observations were transformed to a liability scale (see text).

netic correlations when estimated by assuming either independent residuals $\left(r_{G_{D M}}=-0.11\right)$ or when allowing for correlated residuals $\left(r_{G_{D M}}=-0.13\right)$. Moreover, results from the S-MGS model, which does not use damoffspring relationships, yielded a very similar genetic correlation of -0.15 .

\section{CONCLUSIONS}

Second-parity calving ease in Dutch dairy cattle has a direct heritability of approximately 0.08 , a maternal heritability of approximately 0.04 , a direct-maternal genetic correlation of approximately -0.20 , and a total heritable variance equal to approximately $11 \%$ of phenotypic variance. Results of animal models and S-MGS models were very similar. Transformation of observations to a liability scale did not affect the estimated genetic parameters. The direct-maternal environmental covariance was near zero and had very little effect on the estimated genetic parameters. We conclude, therefore, that environmental dam-offspring covariances are not important in second-parity calving ease in Dutch dairy cattle.

\section{ACKNOWLEDGMENTS}

Data used for this research were kindly provided by the Dutch dairy cattle syndicate (NRS).

\section{REFERENCES}

Bijma, P. 2006. Estimating maternal genetic effects in livestock. J. Anim. Sci. 84:800-806.

Cue, R. I., and J. F. Hayes. 1985. Correlations of various direct and maternal effects for calving ease. J. Dairy Sci. 68:374-381.

Dickerson, G. E. 1947. Composition of hog carcasses as influenced by heritable differences in rate and economy of gain. Iowa Agric. Exp. Stn. Res. Bull. 345:489-524.

Djemali, M., P. J. Berger, and A. E. Freeman. 1987. Ordered categorical sire evaluation for calving ease in Holsteins. J. Dairy Sci. 70:2374-2384.

Falconer, D. S. 1965. Maternal effects and selection response. Pages 763-774 in Genetics Today: Proc. XIth Int. Congr. Genet. Vol. 3. Pergamon, Oxford, UK.

Gilmour, A. R., B. R. Cullis, S. J. Welham, and R. Thompson. 2002. ASReml User Guide Release 1.0. VSN International Ltd., Hemel Hempstead, UK.

Hickey, J. M., M. G. Keane, D. A. Kenny, A. R. Cromie, P. R. Amer, and R. F. Veerkamp. 2007. Heterogeneity of genetic parameters for calving difficulty in Holstein heifers in Ireland. J. Dairy Sci. 90:3900-3908.

Huxley, J. N., and H. R. Whay. 2006. Current attitudes of cattle practitioners to pain and the use of analgesics in cattle. Vet. Rec. 159:662-668.

Koch, R.M. 1972. The role of maternal effects in animal breeding: VI. Maternal effects in beef cattle. J. Anim. Sci. 35:1316-1323.

Koch, R. M., L. V. Cundiff, and K. E. Gregory. 1994. Cumulative selection and genetic chance for weaning or yearling weight or for yearling weight plus muscle score in Hereford cattle. J. Anim. Sci. $72: 864-885$.

Koerhuis, A. N. M., and R. Thompson. 1997. Models to estimate maternal effects for juvenile body weight in broiler chickens. Genet. Sel. Evol. 29:225-249.

Luo, M. F., P. J. Boettcher, L. R. Schaeffer, and J. C. M. Dekkers. 2002. Estimation of genetic parameters of calving ease in first and second parities of Canadian Holsteins using Bayesian methods. Livest. Prod. Sci. 74:175-184.

Manfredi, E., V. Ducrocq, and J. L. Foulley. 1991. Genetic analysis of calving ease in dairy cattle. J. Dairy Sci. 74:1715-1723. 
Mee, J. F. 2007. Prevalence and risk factors for calving ease in dairy cattle: A review. Vet. J. 176:93-101.

Meijering, A., and A. Postma. 1985. Responses to sire selection for dystocia. Livest. Prod. Sci. 13:251-266.

Meyer, K. 1992. Bias and sampling covariances of estimates of variance components due to maternal effects. Genet. Sel. Evol. 24:487509.

Robinson, D. L. 1996. Model which might explain negative correlation between direct and maternal genetic effects. Livest. Prod. Sci. 45:111-122.

Steinbock, L., A. Näsholm, B. Berglund, K. Johansson, and J. Philipsson. 2002. Genetic effects on stillbirth and calving difficulty in Swedish Holsteins at first and second calving. J. Dairy Sci. $86: 2228-2235$.

Thompson, J. R., A. E. Freeman, and P. J. Berger. 1981. Age of dam and maternal effects for calving ease in Holsteins. J. Dairy Sci. 68:1603-1609.

Weller, J. I., and D. Gianola. 1989. Models for genetic analysis of calving ease and calf mortality. J. Dairy Sci. 72:2633-2643.

Weller, J. I., and M. Ron. 1992. Genetic analysis of fertility traits in Israeli Holsteins by linear and threshold models. J. Dairy Sci. $75: 2541-2548$.

Wiggans, G. R., I. Misztal, and C. P. Van Tassell. 2003. Calving ease (co)variance components for a sire-maternal grandsire threshold model. J. Dairy Sci. 86:1845-1848.

Willham, R. L. 1963. The covariance between relatives for characters composed of components contributed by related individuals. Biometrics 19:18-27.

Willham, R. L. 1972. The role of maternal effects in animal breeding III. Biometrical aspects of maternal effects in animal. J. Anim. Sci. 35:1288-1292.

Wright, S. 1934. An analysis of variability in number of digits in an inbred strain of guinea pigs. Genetics 19:506-536.

\section{APPENDIX}

A formal derivation of equation [5] follows from regressing the sum of direct and maternal breeding value on the selection criterion $C$, so that the response to selection equals $R=\Delta \bar{A}_{D}+\Delta \bar{A}_{M}=b_{A_{D}+A_{M}, C}(C-\bar{C})$. In this expression, $(C-\bar{C})$ represents the difference between the mean value of $C$ in the selected parents and the overall mean of $C$, which is the selection differential. By definition, the selection differential is the product of selection intensity and the standard deviation in the selection criterion, $(C-\bar{C})=\iota \sigma_{C}$. The $b_{A_{D}+A_{M}, C}$ represents the regression coefficient of the sum of the direct and maternal breeding value of an individual on the value of its selection criterion. From the definition of a regression coefficient, $b_{A_{D}+A_{M}, C}$ $=\operatorname{Cov}\left(A_{D}+A_{M}, C\right) / \operatorname{Var}(C)$. Combining terms and substituting $\quad A_{D, i}+A_{M, i}=T B V_{i} \quad$ yields $R=\operatorname{Cov}(T B V, C) / \operatorname{Var}(C) \times \iota \sigma_{C}=\operatorname{Cov}(T B V, C) \times \iota / \sigma_{C}$. Multiplying this result by $\sigma_{T B V} / \sigma_{T B V}$ yields equation [5], $R=\iota\left[\operatorname{Cov}(T B V, C) /\left(\sigma_{C} \sigma_{T B V}\right)\right] \sigma_{T B V}=\iota \rho_{M} \sigma_{T B V}$. In this expression, $\rho_{M}=\operatorname{Cov}(T B V, C) /\left(\sigma_{C} \sigma_{T B V}\right)$, which is the correlation between an individual's TBV and the value of its selection criterion, and represents the accuracy of selection for maternally affected traits. 